\title{
On the Operation of Massive MIMO with and without transmitter CSI
}

\author{
Marcus Karlsson and Erik G. Larsson
}

\section{Linköping University Post Print}

\section{Tweet}

N.B.: When citing this work, cite the original article.

Original Publication:

Marcus Karlsson and Erik G. Larsson, On the Operation of Massive MIMO with and without transmitter CSI, 2014, Signal Processing Advances in Wireless Communications (SPAWC), 2014 IEEE 15th International Workshop on, 1-5.

\section{http://dx.doi.org/10.1109/SPAWC.2014.6941305}

C2014 IEEE. Personal use of this material is permitted. However, permission to reprint/republish this material for advertising or promotional purposes or for creating new collective works for resale or redistribution to servers or lists, or to reuse any copyrighted component of this work in other works must be obtained from the IEEE.

\section{http://ieeexplore.ieee.org/}

Postprint available at: Linköping University Electronic Press

http://urn.kb.se/resolve?urn=urn:nbn:se:liu:diva-113564 


\title{
On the Operation of Massive MIMO with and without Transmitter CSI
}

\author{
Marcus Karlsson and Erik G. Larsson
}

\begin{abstract}
The paper considers the issue of activating inactive terminals by control signaling in the downlink in a massive MIMO system. There are two basic difficulties with this. First, the lack of CSI at the transmitter. Second, the short coherence interval, which limits the number of orthogonal pilots in the case of many antennas. The proposed scheme deals with these issues by repeating the transmission over the antennas. We show that this repetition does not affect the spectral efficiency significantly, while making it possible to estimate the channel in a standard way using MMSE. The paper also sheds some light the uplinkdownlink power balance in massive MIMO.
\end{abstract}

\section{BACKGROUND}

Massive MIMO is one of the most promising directions in wireless communications research, and perhaps the only possibility for drastically increasing the spectral efficiency of cellular communications. With massive MIMO, each base station is equipped with a large number of antennas, $M$, operating phase-coherently and serving a large number of terminals, $K$, in the same time-frequency resource; typically, $M$ is in the order of hundreds and $M \gg K \gg 1$. The basic presumption is that the channel is reciprocal, and this reciprocity is exploited by operating in time division duplex (TDD) mode and using uplink pilots to estimate both the uplink and the downlink channels. This channel state information (CSI) is then used to perform multiuser beamforming in the downlink. In several previous studies [1]-[3] the use of maximum ratio combining / maximum ratio transmission (MRC / MRT) processing, also known as conjugate beamforming, is advocated; MRC / MRT processing can be partly performed in a distributed manner, independently at each antenna unit. The use of more advanced processing techniques, zero-forcing (ZF) in particular, does not seem to significantly enhance performance when all impairments, especially pilot contamination, are taken into account [1]-[3]. MRC / MRT processing with TDD operation renders the system completely scalable with $M$. In general, an array/power gain of $\mathcal{O}(M)$ and $\mathcal{O}(\sqrt{M})$ is achievable with perfect and imperfect CSI, respectively [1]. This can potentially make massive MIMO systems very energy efficient [4].

A potential major problem arises when this array gain cannot be harvested, i.e., when the base station needs to contact terminals but does not have CSI. This is the case, for example, when the base station is broadcasting control information or when paging an inactive terminal. It appears

M. Karlsson and E. G. Larsson are with the Department of Electrical Engineering (ISY), Linköping University, 58183 Linköping, Sweden (email: \{marcus.karlsson, erik.g.larsson\}@liu.se).

This work was supported in part by the Swedish Research Council (VR), and ELLIIT.

\begin{tabular}{|c|c|c|}
\hline & $2 \mathrm{GHz}$ Carrier & $6 \mathrm{GHz}$ Carrier \\
\hline Indoor: $1 \mathrm{~m} / \mathrm{s}$ & 7500 & 2500 \\
\hline Outdoor: $100 \mathrm{~km} / \mathrm{h}$ & 270 & 90 \\
\hline Outdoor: $350 \mathrm{~km} / \mathrm{h}$ & 75 & 25 \\
\hline
\end{tabular}

TABLE I

APPROXIMATE LENGTH OF THE COHERENCE INTERVAL (MEASURED IN SYMBOLS) FOR DIFFERENT SCENARIOS, FOR A $200 \mathrm{KHZ}$ BANDWIDTH.

to be widely held that operation of massive MIMO systems without CSI at the transmitter will be very costly.

Against this background, this paper makes the following contributions:

1) We quantitatively examine what operating points are feasible in a massive MIMO system, for some representative scenarios;

2) at these operating points, we examine what performance that can be achieved with and without CSI at the transmitter; and

3) we discuss the choice of transmission strategies for the case of no CSI available at the base station. In particular, we discuss the application of space-time block coding with training-based detection. A new aspect here is that in many cases the length of the coherence interval, $\tau_{c}$ (measured in symbols) is comparable to the number of antennas $M$, so conventional orthogonal training is infeasible.

Conclusions are given in Section IV.

\section{REVIEW OF MASSIVE OPERATION}

\section{A. The coherence interval}

In massive MIMO, all activity takes place within a coherence interval: uplink pilot transmission, uplink payload transmission, and downlink transmission. One can also send supplementary (beamformed) downlink pilots, but these seem to be of limited use in most cases [5]. We denote the coherence time-bandwidth product, measured in symbols, by $\tau_{c}$. Some representative values of $\tau_{c}$ are given in Table I. During the pilot phase, the base station estimates the channels to all terminals using the minimum mean square error (MMSE) estimate.

\section{B. Massive MIMO performance with estimated CSI}

Here, we assume that all terminals are affected by equal path loss and we neglect the effects of shadow fading. 
1) Uplink: In the uplink, the following ergodic sum-rate is achievable with MRC processing in i.i.d. Rayleigh fading:

$$
R_{u}=\left(1-\frac{\tau_{p}}{\tau_{u}}\right) K \log _{2}\left(1+\frac{M \rho_{u}}{\left(K \rho_{u}+1\right)\left(1+\frac{1}{\tau_{p} \rho_{u}}\right)}\right)
$$

The proof of Equation (1) is omitted here, but follows the same steps as for the downlink [2]. In Equation (1), $\tau_{p}$ and $\tau_{u}$ are the number of symbols used for pilots and the total number of symbols available to the uplink in each coherence interval, respectively. Because all terminals should use orthogonal pilots we have $K \leq \tau_{p} \leq \tau_{u} \leq \tau_{c}$. $\rho_{u}$ has the interpretation of normalized transmit power, or the SNR per terminal defined as follows: If one terminal transmits and all others stay silent, $\rho_{u}$ is the SNR observed, on the average (averaged over the small-scale fading), at any of the base station antennas.

We can interpret the different factors in Equation (1) as follows:

- $\left(1-\frac{\tau_{p}}{\tau_{u}}\right)$ is the fraction of the uplink spent on payload data. One can argue if this pilot penalty should solely affect the uplink as it does here. However, this is more of a question about how to divide the coherence interval between uplink and downlink and is not considered in this paper.

- $K$ is the multiplexing gain

- $M \rho_{u}$ is the received useful power

- $K \rho_{u}+1$ represents the received interference plus noise

- $1+\frac{1}{\tau_{p} \rho_{u}}$ is a penalty associated with the reliance on estimated channel state information. This factor is significant if $\tau_{p} \rho_{u} \lesssim \frac{1}{2}$. When this factor cannot be neglected, the only way to compensate for it is to increase $M$. Increasing $\rho_{u}$, even without bound, will not help, since the fraction inside the $\log _{2}(\cdot)$ in Equation (1) tends to $M / K$. Note that increasing $M$ always compensates for poor-quality channel estimates, despite the fact that the number of unknown channel parameters to be estimated grows as $\mathcal{O}(M)$.

Note that the bound in Equation (1) is not as tight as the one derived in [1], but Equation (1) suffices here; it also has the advantage of easier interpretation.

2) Downlink: In the downlink, the ergodic sum-rate is lower bounded by [2]

$$
R_{d}=K \log _{2}\left(1+\frac{M \rho_{d}}{K\left(\rho_{d}+1\right)\left(1+\frac{1}{\tau_{p} \rho_{u}}\right)}\right)
$$

where $\rho_{d}$ is the normalized transmit power for the downlink. $\rho_{d}$ can be seen as the downlink SNR, defined as follows: If all downlink power were radiated from just one of the base station antennas, $\rho_{d}$ would be the average (over the small-scale fading) SNR seen at any of the terminals. In Equation (2), $K$ is the multiplexing gain, $M \rho_{d}$ is the useful beamformed power, $K\left(\rho_{d}+1\right)$ is the received interference at the terminals, and $1+1 /\left(\tau_{p} \rho_{u}\right)$ represents the "penalty" in beamformed power incurred by imperfect channel estimation in the uplink. Like for the uplink, it can be seen that increasing $M$ is the only way to compensate for poor-quality channel estimates.

\section{Uplink-downlink power balance}

What operating points $\left(R_{u}, R_{d}\right)$ are feasible? In order to answer this question, first note that for any given $\left(\rho_{u}, \rho_{d}\right)$ the rates $R_{u}$ and $R_{d}$ can be optimized with respect to $K$ and $\tau_{p}$. Note that the value of $K$ need not be the same in the uplink and the downlink, while this is a necessity for $\tau_{p}$. In the uplink, the sum-rate (Equation (1)) optimized over $K$ and $\tau_{p}$ is given by

$$
R_{u}^{\star}=\max _{\substack{K, \tau_{p} \\ K \leq \tau_{p} \leq \tau_{u}}} R_{u}
$$

and the downlink sum-rate, Equation (2), optimized over $K$ is

$$
R_{d}^{\star}=\max _{\substack{K \\ K \leq \tau_{p}}} R_{d} .
$$

Note that the optimal $\tau_{p}$ is obtained by solving Equation (3) and that this value of $\tau_{p}$ then constitutes the upper bound on $K$ in Equation (4). In our calculations we have chosen $\tau_{u}=\tau_{c} / 2$, which implies that the upper bound on $\tau_{p}$ increases with increased $\tau_{c}$.

The optimization in Equations (3)-(4) results in the contour plots in Figure 1 for different $M$ and $\tau_{c}$ taken from Table I. The horizontal axis shows $\rho_{u}$ and the associated $R_{u}^{\star}$, the vertical axis shows $\rho_{d}$ and each contour curve represents a fixed $R_{d}^{\star}$. The graphs show what $\left(\rho_{u}, \rho_{d}\right)$ pairs are needed to achieve a particular downlink sum-rate.

Note the vertical asymptotes of the curves as $\rho_{u}$ decreases. These asymptotes correspond to operating points where the channel estimates are too poor for the downlink to be able to compensate the poor quality with higher transmit power; if $\rho_{u}$ is low enough, we cannot compensate for it by increasing $\rho_{d}$, only increasing $M$ will help in this case.

The straight lines with slope $45^{\circ}$ in Figure 1 correspond, from the left, to operating points where $10 \rho_{u}=\rho_{d}, \rho_{u}=\rho_{d}$, and $\rho_{u}=10 \rho_{d}$, respectively. Note that when $\rho_{u}=\rho_{d}$, the array emits the same amount of power as each of the terminals, which gives the uplink a substantial power advantage over the downlink. One can argue that this is a good reason to let the uplink pay for the use of pilots, as done here in Equation (1).

The horizontal asymptotes tell us that at some point, increasing $\rho_{u}$ does not improve the downlink rates significantly. Note that in Equation (2), increasing $\rho_{u}$ has the same effect as increasing $\tau_{p}$, since the product $\tau_{p} \rho_{u}$ is the total amount of power spent on pilots. This can be seen in Figure 1, comparing plots with the same number of antennas, but different $\tau_{c}$. Increasing the length of the coherence interval shifts the curves to the left, since $\tau_{p}$ increases. So for the same $\left(\rho_{u}, \rho_{d}\right)$ pair, the downlink rate is increased when $\tau_{c}$ increases. Of course, this increase is also due to the increased number of terminals served. 


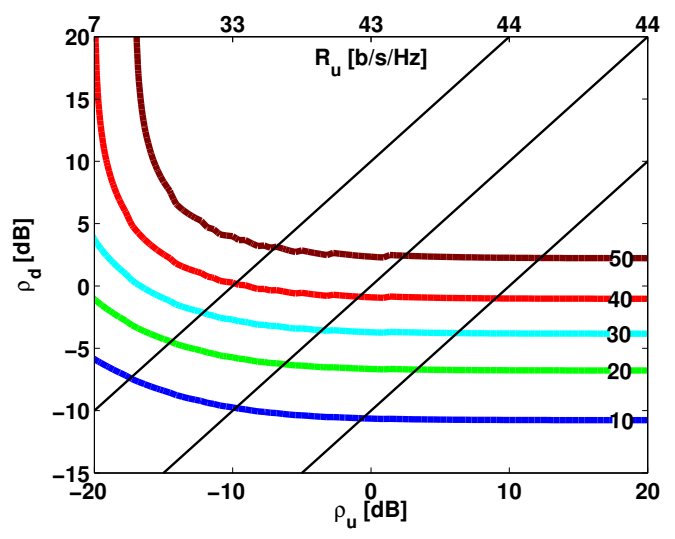

(a) $M=100, \tau_{c}=200$

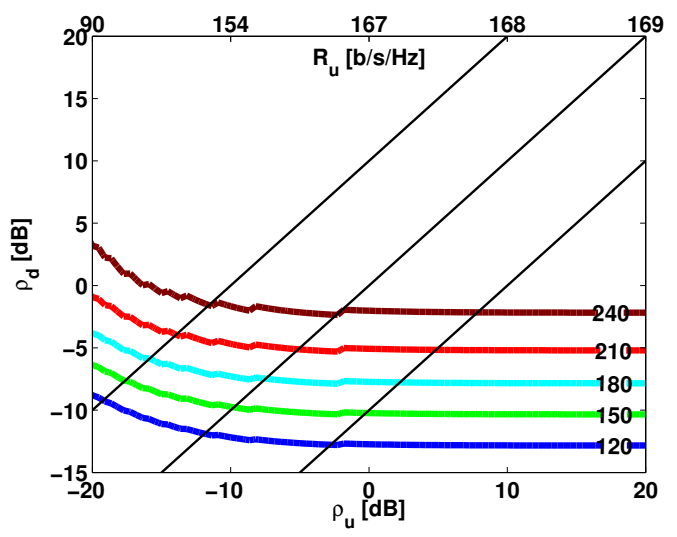

(c) $M=5000, \tau_{c}=200$

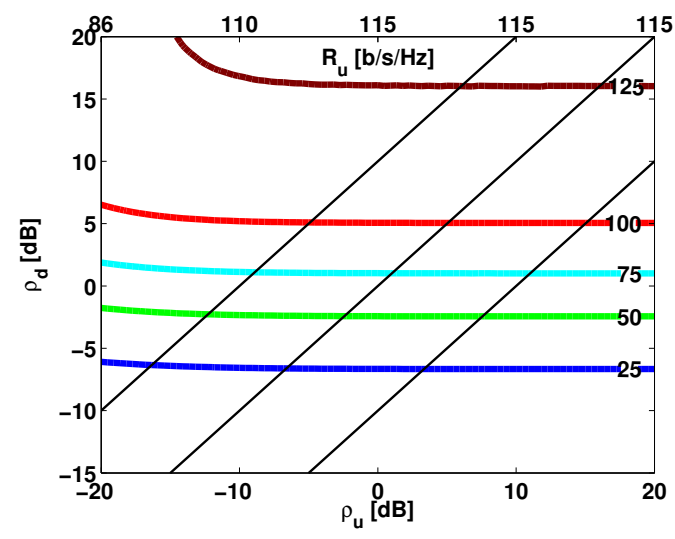

(b) $M=100, \tau_{c}=7500$

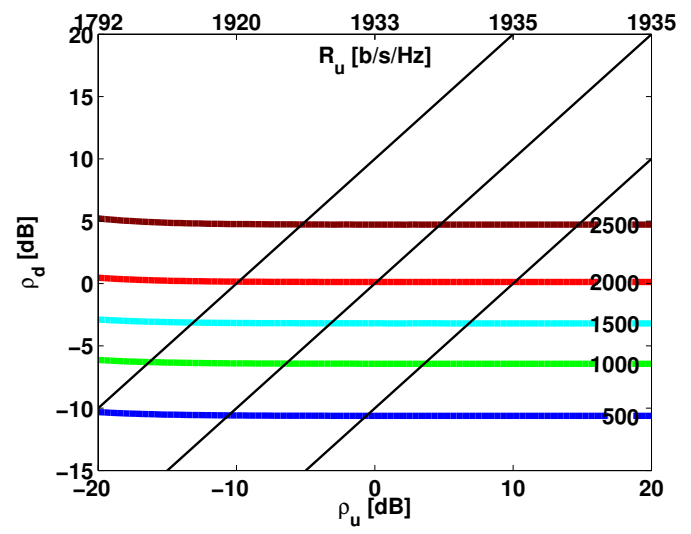

(d) $M=5000, \tau_{c}=7500$

Fig. 1. Contour plots of four different cases (four different combinations of $M$ and $\tau_{c}$ ). The different curves represent fixed downlink sum-rates (see Equation (2)) and show what $\left(\rho_{u}, \rho_{d}\right)$ pairs that give a particular downlink sum-rate. The upper horizontal axis denotes the corresponding uplink sum-rate associated with a particular $\rho_{u}$.

\section{Selection of operating points}

In order to find reasonable operating points for the system and to avoid explicit link budget calculations, a conventional system using single-antenna transmission is taken as a reference. The ergodic rate for this reference system, assuming perfect CSI at the receiver (which is reasonable for SISO if $\left.\tau_{c} \gg 1\right)$, is

$$
R_{S I S O}=\mathrm{E}\left[\log _{2}\left(1+\rho|g|^{2}\right)\right], g \sim \mathcal{C N}(0,1)
$$

where $\rho$ has the interpretation of SNR, just like $\rho_{u}$ and $\rho_{d}$ in previous sections. Now, suppose we would like to deploy massive MIMO in the same scenarios as where the reference system currently provides service. We consider two scenarios:

1) Low SNR: Here, typical values are transmission of $64 \mathrm{~kb} / \mathrm{s}$ over $360 \mathrm{kHz}$ [6], corresponding to approximately $R_{S I S O}=0.2 \mathrm{~b} / \mathrm{s} / \mathrm{Hz}$. This scenario is meant to demonstrate conditions for terminals near the cell edge and in other limited coverage areas, such as basements. Solving Equation (5), assuming Rayleigh fading, we find $\rho \approx 0.1=-10 \mathrm{~dB}$.

2) High SNR: $R_{S I S O}=5$ corresponds to 64-QAM modulation with light coding. For comparison the highest rate in LTE-10 is about $4 \mathrm{~b} / \mathrm{s} / \mathrm{Hz}$ [7]. This corresponds to a scenario where the terminal is close to the base station. Solving Equation (5) gives us $\rho \approx 50 \approx 17 \mathrm{~dB}$.

In reality, most terminals will experience conditions somewhere in between case 1 and 2 above. Hence, a $\rho$ between -10 and $17 \mathrm{~dB}$ appears reasonable.

To give concrete examples of power levels, consider the LTE uplink as reference. In the two scenarios above, LTE supports service using $250 \mathrm{~mW}$ output power over $360 \mathrm{~Hz}$ [6], the transmit power of a terminal in the uplink. If $\rho_{u}=\rho_{d}=0.1=$ $-10 \mathrm{~dB}$ and assuming complete uplink-downlink reciprocity (including identical noise figures at the base station and the terminals), then the base station array emits $250 \mathrm{~mW}$ over 360 $\mathrm{kHz}$, that is, $7 \mathrm{~W}$ over $10 \mathrm{MHz}$. Looking at Figure 1(a), the downlink sum-rate for this $\left(\rho_{u}, \rho_{d}\right)$ pair is close to $10 \mathrm{~b} / \mathrm{s} / \mathrm{Hz}$, near a third of the uplink sum-rate. Here, $K \approx 65$ terminals are served simultaneously. Note that the difference between uplink and downlink would be even larger if the downlink also was punished for the resources spent on pilots. The downlink sum-rate for the high SNR scenario, $\rho_{u}=\rho_{d}=17 \mathrm{~dB}$, is about $80 \mathrm{~b} / \mathrm{s} / \mathrm{Hz}$, whereas the uplink sum-rate is around 45 $\mathrm{b} / \mathrm{s} / \mathrm{Hz}$. Looking at scenarios 1 and 2 above, moving from low to high SNR increases the downlink sum-rate much more than 


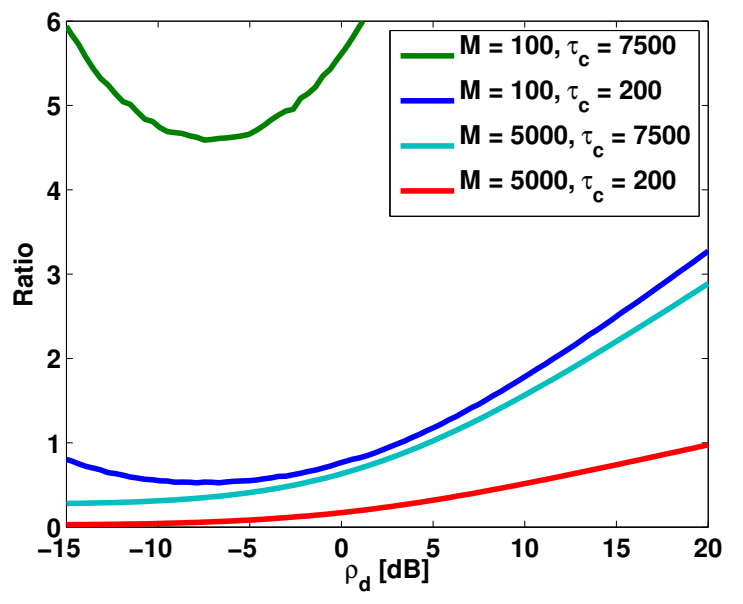

Fig. 2. The ratio between Equation (7) and the rate per terminal given by Equation (4) divided by the number of terminals served, i.e., $\frac{s_{d} K^{\star}}{R_{d}^{\star}}$. The ratio is shown for the same four scenarios as in Figure 1.

the uplink sum-rate. In the high SNR case this means that, for a fixed $\rho_{d}$ and a lower bound on "tolerable" downlink sumrate, $\rho_{u}$ can be decreased by a large amount, before this lower bound is reached; this is not true for the low SNR case.

\section{Operation Without TransmitTer CSI}

We now consider the case that the base station does not have any CSI. Then, the only reasonable transmit strategy is to spread the power omnidirectionally, that is, if $\boldsymbol{x}$ is an $M$ vector of emitted samples, then

$$
\mathrm{E}\left[\boldsymbol{x} \boldsymbol{x}^{\mathrm{H}}\right]=\frac{\rho_{d}}{M} \boldsymbol{I}
$$

The normalization in Equation (6) ensures that any terminal will see an SNR of $\rho_{d}$, and that the array emits the same total power as in Section II-C. If $\boldsymbol{g}$ represents the channel between the array and a terminal, then with Equation (6), the following ergodic rate can be achieved:

$$
s_{d} \triangleq \mathrm{E}\left[\log _{2}\left(1+\frac{\rho_{d}}{M}\|\boldsymbol{g}\|^{2}\right)\right] \approx \log \left(1+\rho_{d}\right),
$$

if $\boldsymbol{g}$ is known at the terminal and $M \gg 1$. Note that the rate in Equation (7) is actually comparable to the rate per served terminal when beamforming is used, that is, $R_{d}^{\star}$ in Equation (4) divided by the optimal value of $K$, say $K^{\star}$. Figure 2 shows the ratio between $s_{d}$ in Equation (7) and $R_{d}^{\star} / K^{\star}$ in Equation (4), for the same four cases as in Figure 1.

First, comparing the same antenna array, but with different length of the coherence interval, we see that the curve corresponding to the scenario with large $\tau_{c}$ lies strictly above the curve corresponding to small $\tau_{c}$. Even though the sum-rate increases with $\tau_{c}$ (Figure 1), we see that the rate per terminal is lower. The increase in sum-rate, when CSI is available, mainly comes from the ability to serve more users, not increased individual rates.

Second, comparing different values of $M$ shows that increasing $M$ provides each terminal with a higher rate, as well as increases the overall sum-rate. Even though the rate per terminal is similar for $M=100, \tau_{c}=200$ and $M=$ $5000, \tau_{c}=7500$ for $\rho_{u}=\rho_{d}$ close to zero, the downlink sum-rate of the latter is close to 50 times higher.

However, we stress that Equation (7) is actually an upper bound on the rate because of the assumption of channel knowledge at the terminal. We will relax this assumption shortly.

\section{A. The finite coherence interval problem}

In principle, space-time block codes (STBCs) [8] can achieve rates close to the rate in Equation (7). The difficulty, however, is that to decode the STBCs, the terminal needs to know the channel $\boldsymbol{g}$. Transmission of pilots that facilitate learning of the channel by the terminals will consume at least $M$ out of the $\tau_{c}$ symbols in the coherence interval. This is due to the fact that optimal training consists of sending orthogonal pilots from each antenna at the base station [8, lemma 9.1]. If $M$ is comparable to $\tau_{c}$, then most of the coherence interval needs to be used for pilots and if $M>\tau_{c}$, the whole process is impossible. This calls for a different, suboptimal (in terms of pilots) transmission scheme to estimate the channels.

\section{B. Repeated transmission}

Consider restricting all emitted vectors to be of the form

$$
\boldsymbol{x}=\left[\begin{array}{c}
\boldsymbol{x}^{\prime} \\
\boldsymbol{x}^{\prime} \\
\vdots \\
\boldsymbol{x}^{\prime}
\end{array}\right] \in \mathcal{C}^{M \times 1}, \boldsymbol{x}^{\prime} \triangleq\left[\begin{array}{c}
x_{1}^{\prime} \\
\vdots \\
x_{M^{\prime}}^{\prime}
\end{array}\right] \in \mathcal{C}^{M^{\prime} \times 1}
$$

for some $M^{\prime}$ which is a factor of $M$, say $M=r M^{\prime}$. That is, $r$ can be seen as the repetition factor, how many times each symbol is repeated over the $M$ antennas; $r=1$ corresponds to no repetition. The ergodic capacity for this repeated scheme, assuming coding over frequency and perfect CSI at the receiver, is

$$
\mathrm{E}\left[\log _{2}\left(1+\boldsymbol{g}^{\mathrm{H}} \boldsymbol{Q g}\right)\right],
$$

where $\boldsymbol{Q}=\mathrm{E}\left[\boldsymbol{x} \boldsymbol{x}^{\mathrm{H}}\right]$. If $\boldsymbol{x}^{\prime}$ in Equation (8) satisfies $\mathrm{E}\left[\boldsymbol{x}^{\prime} \boldsymbol{x}^{\mathrm{H}}\right]=$ $\frac{\rho_{d}}{M} \boldsymbol{I}$, then $\operatorname{Tr}(\boldsymbol{Q})=\rho_{d}$, so the emitted power is the same as assumed for Equation (6). The repetition in Equation (8) entails a loss of diversity, but the loss is not major, as illustrated in Figure 3.

For $r \lesssim 10$ the loss associated with the repeated scheme in Equation (8) is very marginal, only a few percent. The reason for this is that the spatial diversity provided by the $M$ antennas does not affect the spectral efficiency that much, because of the frequency diversity. Even when repeating, the array still provides an array gain. Note that Figure 3 is for MISO channels with perfect CSI at the receiver.

\section{Practical implementation}

In the light of the discussion in Section III-B, in particular, Figure 3 we design a STBC code for the repeated transmission scheme. Assume the system is allowed $\tau_{p}$ symbols for 


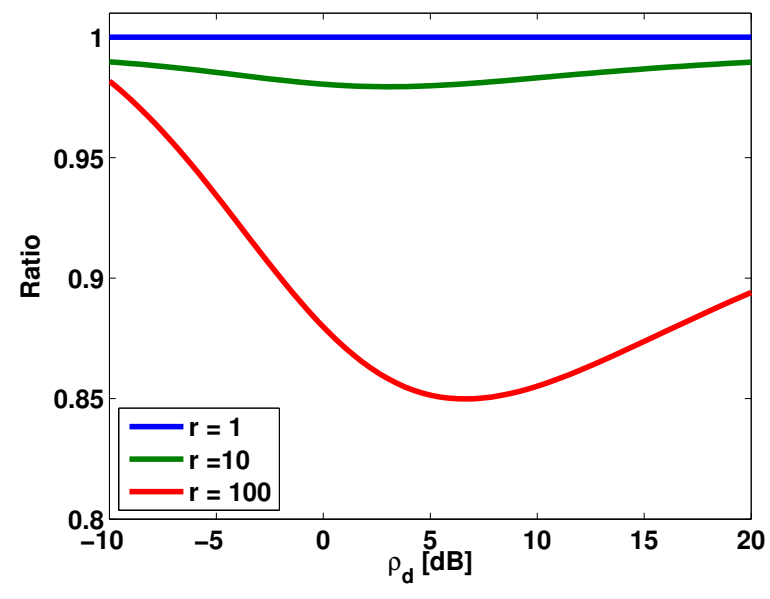

Fig. 3. Comparison of ergodic spectral efficiency of two MISO systems with perfect CSI at the receiver, one with repeated symbols as in Equation (8), the other one with independent symbols.

pilots, and that $M>\tau_{p}$. The pilot matrix is denoted by $\boldsymbol{X}_{p} \in \mathcal{C}^{M \times \tau_{p}}$. If the received signal, channel matrix and noise are denoted by $\boldsymbol{Y}_{p} \in \mathcal{C}^{K \times \tau_{p}}, \boldsymbol{H} \in \mathcal{C}^{K \times M}$, and $\boldsymbol{W} \in \mathcal{C}^{K \times \tau_{p}}$, respectively, we can write

$$
\boldsymbol{Y}_{p}=\boldsymbol{H} \boldsymbol{X}_{p}+\boldsymbol{W}
$$

where $K$ is the number of terminals. Noting that $\boldsymbol{X}_{p}$ is tall, we see that regular ML/LS estimation of the channel $\boldsymbol{H}$ cannot be done, since $\left(\boldsymbol{X}_{p} \boldsymbol{X}_{p}^{\mathrm{H}}\right)^{-1}$ does not exist. To get around this, consider dividing $\boldsymbol{X}_{p}$ in a similar way as in Equation (8), with the repetition factor $r$ chosen such that $M^{\prime} \leq \tau_{p}$. This gives the following equivalent channel:

$$
\boldsymbol{Y}_{p}=\sum_{i=1}^{r} \boldsymbol{H}_{i} \boldsymbol{X}+\boldsymbol{W}=\boldsymbol{G} \boldsymbol{X}+\boldsymbol{W}
$$

where we have defined $\boldsymbol{G} \triangleq \sum_{i=1}^{r} \boldsymbol{H}_{i}$, with $\boldsymbol{H}_{i}$ being the $i$ :th $K \times M^{\prime}$ submatrix of $\boldsymbol{H}$, i.e., $\boldsymbol{H}=\left[\boldsymbol{H}_{1} \boldsymbol{H}_{2} \ldots \boldsymbol{H}_{r}\right]$. Using the [8, lemma 9.1], the optimal $\boldsymbol{X}$ for the equivalent channel $\boldsymbol{G}$ is to choose $\boldsymbol{X}$ orthogonal.

From Equation (11) the $K$ terminals can estimate the channel $G$ with any standard technique, such as LS or MMSE. For the latter, the estimated channel is given by

$$
\hat{\boldsymbol{G}}=\boldsymbol{Y}_{p}\left(\boldsymbol{X}^{\mathrm{H}} \boldsymbol{X}+\boldsymbol{I}\right)^{-1} \boldsymbol{X}^{\mathrm{H}}=\boldsymbol{Y}_{p} \boldsymbol{X}^{\mathrm{H}}\left(\boldsymbol{X} \boldsymbol{X}^{\mathrm{H}}+\boldsymbol{I}\right)^{-1},
$$

if we assume that the noise components are $\mathcal{C N}(0,1)$. Furthermore, if we let $\rho_{p}$ denote the normalized transmit power for the pilots, $\boldsymbol{X} \boldsymbol{X}^{\mathrm{H}}=\rho_{p} \boldsymbol{I}$, then we can write Equation (12) as

$$
\hat{\boldsymbol{G}}=\frac{\rho_{p} \boldsymbol{G}}{\rho_{p}+1}+\frac{\boldsymbol{W} \boldsymbol{X}_{p}^{\mathrm{H}}}{\rho_{p}+1} .
$$

Note that the terminals are completely autonomous and each terminal only knows the channel from the $M^{\prime}$ "artificial" base station antennas to its own antenna. This means that, although MMSE estimation of the effective channel can be done at each terminal, methods like MMSE or ZF cannot be used for detecting the data symbols.

There is a fundamental trade-off between a good channel estimate and the data rate following this estimation, this tradeoff can be directly related to the repetition factor $r$. Large $r$, i.e., high degree of repetition, implies more measurements per estimated parameter, since the dimension of the effective channel decreases with increasing $r$. This gives a better estimate of the effective channel, but limits the successive data transfer because the effective channel to the terminal is an $M^{\prime} \times 1$ channel, hence diversity is reduced, cf. Figure 3 . The payload data following the pilots has to be repeated in the same manner as the pilot matrix for the channel estimate to be useful. This means that the effective data rate decreases somewhat as $r$ grows, due to the same data being transferred over several of the antennas. We stress that this repetition is spatial, over the antennas, not in time.

\section{CONCLUSION}

The issue with transmission of control signaling without CSI at the base station does not seem to be as difficult as it appears at first sight. The achievable rates per terminal with and without beamforming are comparable in many scenarios of interest. For the case without CSI at the base station, when there is enough coding over the frequency domain,repeating the transmission over groups of antennas results in a relatively minor loss of ergodic rate. At the same time, such repetition yields an effective channel that can be trained using a pilot matrix of a much smaller dimension, hence consuming less of the coherence interval. The repetition factor may be chosen on a case by case basis.

Future work will include investigating the optimal structure of the pilot matrix, as well as the data used for the control signaling. In particular, optimal detection at the terminals as well as achievable rates for the scenario with completely autonomous terminals will be studied.

\section{REFERENCES}

[1] H. Q. Ngo, E. Larsson, and T. Marzetta, "Energy and spectral efficiency of very large multiuser MIMO systems," IEEE Transactions on Communications, vol. 61, no. 4, pp. 1436-1449, April 2013.

[2] H. Yang and T. Marzetta, "Performance of conjugate and zero-forcing beamforming in large-scale antenna systems," IEEE Journal on Selected Areas in Communications, vol. 31, no. 2, pp. 172-179, February 2013.

[3] — " "Total energy efficiency of cellular large scale antenna system multiple access mobile networks," in IEEE Online Conference on Green Communications (GreenCom), Oct 2013, pp. 27-32.

[4] E. Björnson, L. Sanguinetti, J. Hoydis, and M. Debbah, "Designing multiuser MIMO for energy efficiency: When is massive MIMO the answer?" CoRR, vol. abs/1310.3843, 2013.

[5] H. Q. Ngo, E. Larsson, and T. Marzetta, "Massive MU-MIMO downlink TDD systems with linear precoding and downlink pilots," in 51st Annual Allerton Conference on Communication, Control, and Computing, Oct 2013, pp. 293-298.

[6] D. H. Holma and D. A. Toskala, LTE for UMTS - OFDMA and SC-FDMA Based Radio Access. Wiley Publishing, 2009.

[7] E. Dahlman, S. Parkvall, and J. Sköld, 4G: LTE/LTE-Advanced for Mobile Broadband. Kidlington, Oxford, UK: Academic Press, 2013.

[8] E. G. Larsson and P. Stoica, Space-Time Block Coding for Wireless Communications. New York, NY, USA: Cambridge University Press, 2003. 